\title{
A comparison of stick and slip contact conditions for a coated sphere compressed by a rigid flat
}

\author{
Shai RONEN, Roman GOLTSBERG*, Izhak ETSION \\ Department of Mechanical Engineering, Technion, Haifa 32000, Israel \\ Received: 28 April 2017 / Revised: 09 June 2017 / Accepted: 19 June 2017 \\ (C) The author(s) 2017. This article is published with open access at Springerlink.com
}

\begin{abstract}
A finite element analysis is used to study the elastic-plastic contact of a coated sphere compressed by a rigid flat under the stick contact condition. This was done for a wide range of hard coating material properties and coating thicknesses. A comparison with the slip contact condition is presented in terms of the critical contact parameters (at yield inception) and plasticity evolution. Empirical expressions are provided for critical interferences of the first and second yield inceptions, in the coating and on the substrate side of the interface, respectively. An expression is also provided for the dimensionless coating thickness for optimal resistance to plasticity under the stick contact condition. Additionally, the relations between different contact parameters in the elastic-plastic regime are presented. In general, it was found that the contact condition has a negligible effect on the contact parameters, except for phenomena occurring close to the contact area.
\end{abstract}

Keywords: contact mechanics; coatings; stick; elastic-plastic

\section{Introduction}

In many engineering applications, mechanical components are in contact and slide relative to each other. Coatings are commonly used in these cases to improve tribological performance, for instance, the enhancement of electrical and thermal conductivity and reduction of friction and wear. These improvements can be seen in a variety of applications, including the automotive [1], electronics [2-5], optical storage disk [6], and cutting tool industries [7]. Despite the wide usage of coatings in many industries, the selection of coating thickness and mechanical properties is still done mainly by trial and error, which wastes funds and time. Optimization of coating properties for specific applications, based on a scientific theory, is still lacking.

The real surfaces of mechanical components are rough and can be modeled as clusters of asperities. The interaction between such rough surfaces occurs at the summits of their contacting asperities $[8,9]$.
Hence, in order to gain a good understanding of such interactions, it is necessary to first study the contact mechanics of a single asperity. This may then be extended to a contact model for a rough surface with a statistical distribution of asperities. There are two approaches to study the contact mechanics of a single asperity, namely flattening and indentation [10]. Most of the theoretical studies on spherical coated contacts that have been published so far focus on the indentation of coated flat substrates by an uncoated spherical indenter [11-17]. This approach is mainly used to characterize the mechanical properties of coatings. However, in order to investigate the optimum tribological performance, flattening, rather than indentation, is the better choice. This is because when tangential loading is applied, indentation is associated with high abrasive friction and wear that results from plowing, compared to the mild adhesive friction and wear of flattening.

Historically, the contact of a single uncoated spherical

* Corresponding author: Roman GOLTSBERG, E-mail: goltsberg.roman@gmail.com 


\begin{tabular}{|c|c|c|c|}
\hline \multicolumn{4}{|c|}{ Nomenclature } \\
\hline$A$ & contact area & $\varepsilon_{\mathrm{y}}$ & yield strain $Y / E$ \\
\hline$a$ & contact radius & $\sigma_{\text {eq }}$ & equivalent von Mises stress \\
\hline$C_{\mathrm{v}}$ & $1.234+1.256 v$ & $\omega$ & interference in slip \\
\hline$E$ & Young's modulus & \multirow{2}{*}{\multicolumn{2}{|c|}{ Subscripts }} \\
\hline$L$ & load in stick & & \\
\hline$P$ & load in slip & $\mathrm{c}$ & critical value \\
\hline$R$ & radius of the spherical substrate (inner & $\mathrm{c} 1$ & first critical value \\
\hline & radius of the coating) & c2 & second critical value \\
\hline$r$ & $\begin{array}{l}\text { radial distance from the center of contact } \\
\text { interface }\end{array}$ & C_CO & $\begin{array}{l}\text { critical value of solid sphere made of the } \\
\text { coating material }\end{array}$ \\
\hline$t$ & coating thickness & \multirow[t]{2}{*}{ c_su } & critical value of solid sphere made of the \\
\hline Y & yield strength & & substrate material \\
\hline$z$ & vertical distance from the center of contact & $\mathrm{CO}$ & coating \\
\hline & interface & $\mathrm{p}$ & corresponds to peak value \\
\hline$\delta$ & interference in stick & st & stick contact condition \\
\hline$v$ & Poisson's ratio & su & substrate \\
\hline
\end{tabular}

asperity was studied by Hertz in 1882 (see, e.g., Ref. [18]). Hertz assumed frictionless (slip) contact of perfectly elastic solids and provided relations between different contact parameters, such as contact load, contact area, and interference. Kogut and Etsion [19] extended Hertz's study for a homogeneous frictionless spherical contact into the elastic-plastic regime, using a finite element method (FEM). By properly normalizing the contact parameters, simple empirical relations were achieved. Goodman [20] was probably the first to address the elastic spherical contact problem under the stick contact condition, and present an analytical solution. Goodman assumed a Hertzian distribution of contact pressure and introduced a tangential stress distribution over the contact area to account for the stick contact condition. A more accurate analysis of indentation under the stick condition was published by Spence [21], who numerically solved the shear stresses and pressure distribution over the contact area and calculated the total compressive load. It follows from the results obtained by Spence, that for small values of Poisson's ratio the influence of the shear stresses on the contact area is significant. Brizmer et al. [22] studied, using FEM, the effect of the contact conditions and material properties on the elasticity terminus of a spherical contact under stick and slip contact conditions. It was found that for small values of the Poisson's ratio, the behavior for stick is much different than that for slip, which was attributed in Ref. [22] to the high tangential stresses at the contact interface in the former case. The critical values (at yield inception) of interference and load for the stick condition are lower than the corresponding values for the slip condition, and the location of yield inception is closer to the contact area for the stick condition. At higher values of Poisson's ratio, the tangential stresses under the stick contact condition are lower and the critical values for stick and for slip become comparable. Brizmer et al. [23] extended the study in Ref. [22] into the elastic-plastic regime. The results, presented in Refs. [19, 23], were experimentally verified by Overcharenko et al. [24].

Goltsberg et al. [25] investigated the plastic yield inception of a coated sphere pressed by a rigid flat under the slip contact condition. An optimum value of the coating thickness for the best resistance to the onset of plasticity was found, and a potential weakening effect at very small coating thicknesses was detected. 
Similar behavior was found for indentation of a coated flat by a rigid sphere in Ref. [26]. The existence of the weakening effect was experimentally verified by Huang et al. [27]. This weakening effect was extensively studied and characterized in Ref. [28]. Further studies by Goltsberg and Etsion [29,30] provided universal relations for various contact parameters in the elastic regime of a coated spherical contact. Sun et al. [31] used FEM to analyze the plastic deformation of a coated spherical contact for the indentation approach. They presented results for relatively thin coatings, where yielding first occurred on the substrate side of the substrate/coating interface followed by a second yielding on the coating side of the interface at higher indentation depth. In Refs. [32, 33], it was found for some specific cases of indentation that the first yield inception occurred within the coating. In these cases, increasing the indentation depth beyond the first yield caused a second yield inception on the substrate side of the interface. All these studies [31-33] examined a limited number of mechanical and geometrical properties of the coating and the substrate materials. Chen et al. [34] investigated the elastic-plastic contact of a coated sphere compressed by a rigid flat under the slip contact condition. This was done for relatively thick coating thicknesses, where the first onset of plasticity occurred in the coating and the second on the substrate side of the interface. Empirical expressions were provided for the critical interferences of the first and second yield inceptions as functions of the coating thickness and material properties. It was shown that a combination of a thick coating and small moduli ratio $E_{\mathrm{co}} / E_{\mathrm{su}}$ is beneficial for protecting against substrate yielding.

As can be seen from the above literature review, the flattening of a coated spherical contact has been modeled so far only under the slip contact condition. Hence, the main goal of the present study is to investigate the coated spherical contact under the stick contact condition for a wide range of coating and substrate material properties. The stick contact condition is necessary to study the combined, normal, and tangential loading, which is essential for future research on the effect that coating properties have on friction and wear.

\section{Theoretical background}

Figure 1 schematically presents a coated sphere system before loading (Fig. 1(a)) and, in contact, after loading (Fig. 1 (b)) by a rigid flat. It consists of a solid spherical substrate of radius $R$, which is covered by a coating layer of thickness $t$. The application of a load, $P$, results in a corresponding interference, $\omega$, of the rigid flat, and the formation of a contact area with radius $a$, owing to the deformation of the coated sphere. When the coating material is identical to the substrate material, Fig. 1 merely describes a homogeneous uncoated case.

Brief descriptions of a homogeneous spherical contact model under the stick contact condition and a coated sphere under the slip contact condition are provided in the following sections.

\subsection{Yield inception of a homogeneous sphere under the stick contact condition [22]}

The critical interference $\delta_{\mathrm{c}}$ and critical load $L_{\mathrm{c}}$ at yield inception under the stick contact condition were given

(a)
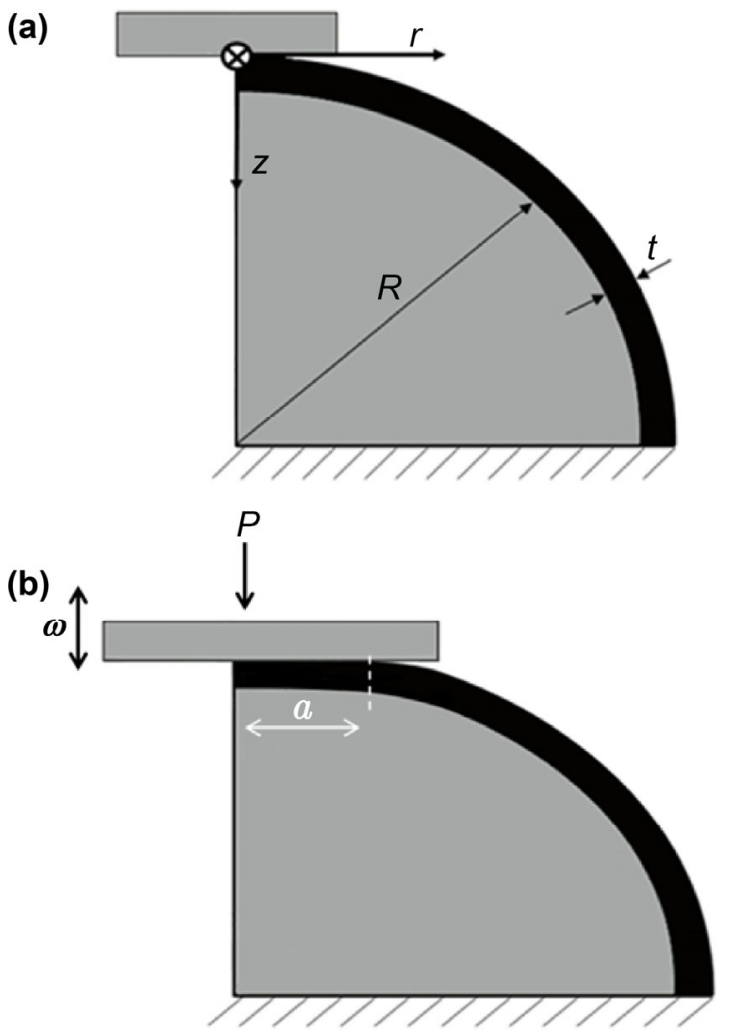

Fig. 1 Schematic of a coated deformable sphere pressed by a rigid flat, before loading (a) and in contact after loading (b) (taken from Ref. [30]). 
by Brizmer et al. [22] in the following form:

$$
\begin{aligned}
& \frac{\delta_{\mathrm{c}}}{\omega_{\mathrm{c}}}=6.82 v-7.83\left(v^{2}+0.0586\right) \\
& \frac{L_{\mathrm{c}}}{P_{\mathrm{c}}}=8.88 v-10.13\left(v^{2}+0.089\right)
\end{aligned}
$$

where $\omega_{\mathrm{c}}$ and $P_{\mathrm{c}}$ are the corresponding critical parameters under perfect slip are given by

$$
\begin{gathered}
\omega_{\mathrm{c}}=\left[C_{\mathrm{v}} \frac{\pi\left(1-v^{2}\right)}{2}\left(\frac{Y}{E}\right)\right]^{2} R \\
P_{\mathrm{c}}=\frac{\pi^{3} Y}{6} C_{\mathrm{v}}{ }^{3}\left[R\left(1-v^{2}\right)\left(\frac{Y}{E}\right)\right]^{2}
\end{gathered}
$$

It was found that for ductile materials $(0.2<v<0.5)$, the yield inception always occurs at a single point on the axis of symmetry. As can be seen in Fig. 2, for small values of $v$, the critical interference and the critical load in the stick contact condition are considerably smaller than their corresponding parameters in the slip contact condition. This is because of high tangential stresses at the contact interface under stick. For increasing values of the Poisson's ratio, the tangential stresses, under the stick contact condition, decrease and the critical parameters become comparable to those in slip.

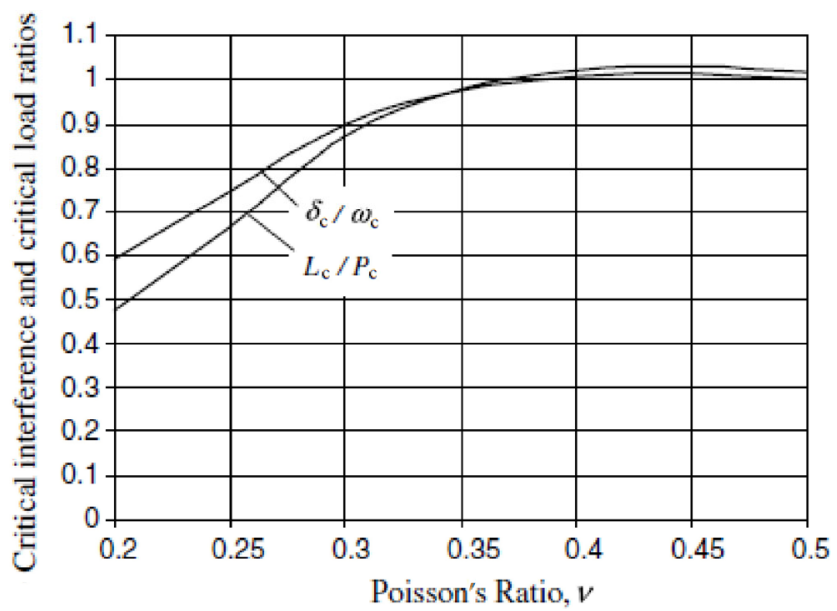

Fig. 2 The ratios of the critical interference, $\delta_{\mathrm{c}} / \omega_{c}$, and critical load, $L_{\mathrm{c}} / P_{\mathrm{c}}$, for yield inception of ductile materials in full stick over perfect slip [22].

\subsection{Coated sphere under the slip contact condition} $[25,34]$

Figure 3, taken from Ref. [25], presents the dimensionless critical load $P_{\mathrm{c}} / P_{\mathrm{c}_{-} \text {co }}$ of a coated sphere as a function of the dimensionless coating thickness $t / R$, for different $P_{c_{-} \text {co }} / P_{\mathrm{c}_{\_} \text {su }}$ ratios. $P_{\mathrm{c}_{-} \text {co }}$ and $P_{\mathrm{c}_{-} \text {su }}$ are the critical loads at yield inception for a homogeneous sphere made of the coating and substrate materials, respectively [25]. As can be seen, the highest resistance to the onset of plasticity for each curve is associated with a certain value of the dimensionless coating thickness. This coating thickness depends on material properties, and it was given in Ref. [25] in the form

$$
\left(\frac{t}{R}\right)_{\mathrm{p}}=2.824\left(\frac{E_{\mathrm{su}}}{Y_{\mathrm{su}}}\right)^{-1.014}\left(\frac{P_{\mathrm{c} \_ \text {co }}}{P_{\mathrm{c} \_ \text {su }}}\right)^{0.536}
$$

where

$$
\frac{P_{\mathrm{c}_{-} \mathrm{co}}}{P_{\mathrm{c} \_ \text {su }}}=\left(\frac{C_{\mathrm{v}_{\mathrm{c}} \mathrm{co}}}{C_{\mathrm{v}_{\mathrm{s}} \mathrm{su}}}\right)^{3} \frac{\left(1-v_{\mathrm{co}}^{2}\right.}{\left(1-v_{\mathrm{su}}^{2}\right)}\left(\frac{Y_{\mathrm{co}}}{Y_{\mathrm{su}}}\right)^{3}\left(\frac{E_{\mathrm{su}}}{E_{\mathrm{co}}}\right)^{2}
$$

When the substrate and coating material have the same Poisson's ratio, Eq. (5) can also be written as (provided in Ref. [34])

$$
\left(\frac{t}{R}\right)_{\mathrm{p}}=2.824\left(\frac{E_{\mathrm{co}}}{E_{\mathrm{su}}}\right)^{0.536}\left(\frac{E_{\mathrm{co}}}{Y_{\mathrm{co}}}\right)^{-1.608}\left(\frac{E_{\mathrm{su}}}{Y_{\mathrm{su}}}\right)^{0.594}
$$

It should be noted here that both Eqs. (5) and (7) were obtained for a relatively small range of $E_{\mathrm{co}} / E_{\mathrm{su}}$ values, between 1 and 4.5 .

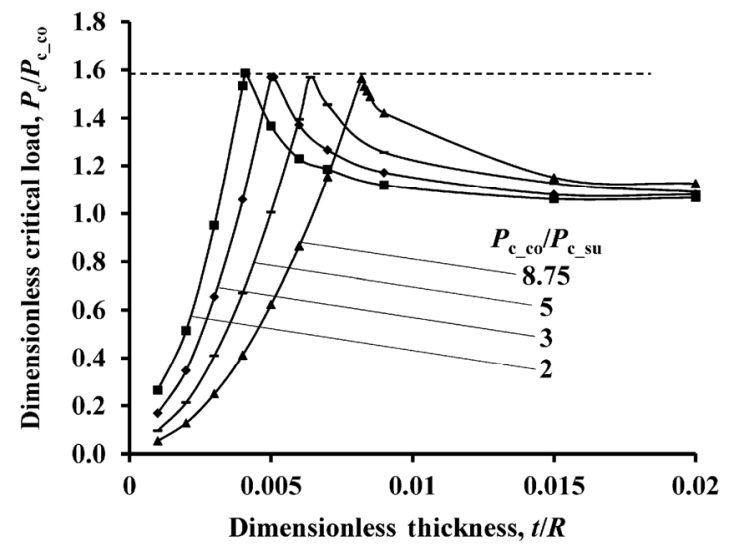

Fig. 3 Dimensionless critical load as a function of the dimensionless thickness $t / R$, for different values of critical loads ratios [25]. 
It was found that for very thin coatings, where $t / R$ is much smaller than $(t / R)_{p}$, yielding initiates within the substrate (location 1 in Fig. 4). When $t / R$ is higher, but still lower than $(t / R)_{\mathrm{p}}$, yield inception occurs on the substrate side of the interface (location 2 in Fig. 4). When $t / R$ is higher than $(t / R)_{p}$, the yield inception occurs slightly below the contact area within the coating (location 3 in Fig. 4). Although the analysis in Ref. [25] was performed mainly for $v=0.32$, it was indicated that for a coated sphere under the slip contact condition, the effect of varying the Poisson's ratio is negligible (less than 10\%).

Chen et al. [34] showed that for $t / R>(t / R)_{p}$, as the interference increases above that corresponding to the first yield in the coating, $\omega_{\mathrm{c} 1}$, a second yield occurs in the substrate at a second critical interference, $\omega_{\mathrm{c} 2}$. The empirical expressions for these two critical interferences were given in Ref. [34] as follows:

$$
\begin{aligned}
& \frac{\omega_{\mathrm{c} 1}}{\omega_{\mathrm{c} \_\mathrm{co}}}=1+3.78\left(\frac{t}{R}\right)^{-1.29}\left(\frac{E_{\mathrm{co}}}{E_{\mathrm{su}}}-1\right)^{0.811}\left(\frac{E_{\mathrm{co}}}{Y_{\mathrm{co}}}\right)^{-1.3} \\
& \frac{\omega_{\mathrm{c} 2}}{\omega_{\mathrm{c} \_\mathrm{co}}}=0.25\left(\frac{t}{R}\right)^{1.34}\left(\frac{E_{\mathrm{co}}}{E_{\mathrm{su}}}\right)^{-0.14}\left(\frac{E_{\mathrm{co}}}{Y_{\mathrm{co}}}\right)^{2}\left(\frac{E_{\mathrm{su}}}{Y_{\mathrm{su}}}\right)^{-0.66}
\end{aligned}
$$

\section{The finite element model}

The commercial FE package ANSYS 14.0 was used to solve the contact problem using an axisymmetric $2 \mathrm{D}$ model, as presented in Fig. 5. The nodes on the bottom

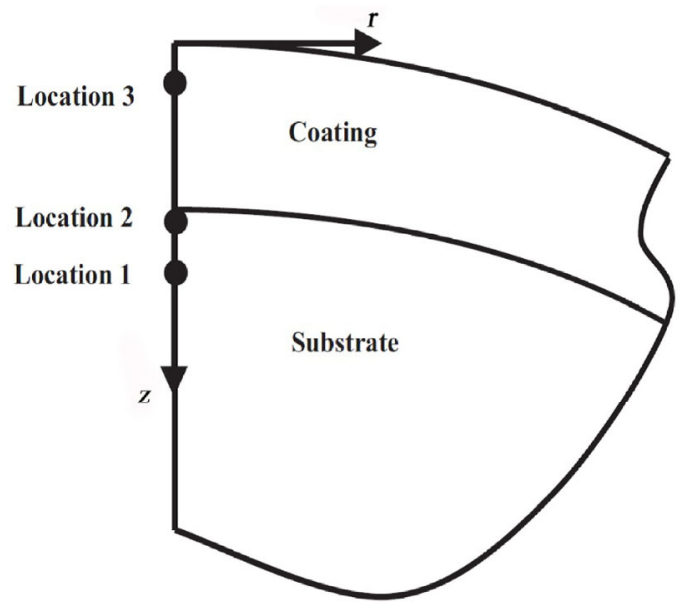

Fig. 4 Typical locations of yield inception in a coated sphere compressed by a rigid flat [25].

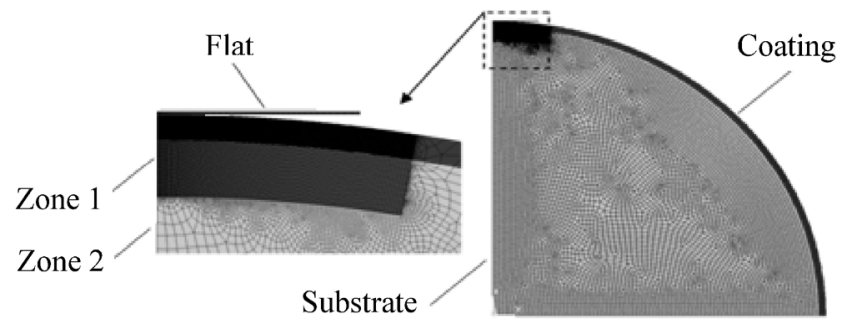

Fig. 5 The finite element mesh of a coated sphere.

of the system were constrained in both the axial and radial direction. To simulate the rigidity of the flat, its Young's modulus was chosen to be seven orders of magnitude higher than that of the coating and the substrate. An eight-node axisymmetric quadrilateral solid element (PLANE183) was used for the coating, substrate, and flat. A three-node contact element (CONTA175) was used on the outer surface of the coating, and a target element (TARGE169) was used at the bottom of the rigid flat. The mesh of the coated sphere was divided into two different mesh density zones (see Fig. 5). Zone 1, with a uniform fine density mesh, contains the part of the coated system where plasticity evolves in both the coating and the substrate. In this zone, the typical length of the elements for any radius $R$ was in the order of $0.001 R$. The width of zone 1 was chosen to be 15 times the width of the contact radius for the case of the Hertz solution. Zone 2 had a gradually coarser mesh with increasing distance from zone 1 . The total number of nodes in zones 1 and 2 was about 50,000.

The following main assumptions are adopted for the model [34]:

1 . The coating is perfectly bonded to the substrate.

2. The coating and substrate materials are homogenous.

3. The coating and substrate are free of residual stresses.

4. The Poisson's ratio for the coating and substrate materials are constant and equal, i.e., $v_{\mathrm{co}}=v_{\mathrm{su}}=0.32$.

The only different assumption compared to that in Ref. [34] is that a stick contact condition prevails between the coating and the rigid flat.

The substrate and coating materials were defined as elastic-plastic with linear isotropic hardening and a tangent modulus of $2 \%$ of their respective Young's modulus, similarly to the definition in Ref. [34]. This hardening enables to compare the results of this paper 
to those published for the slip contact condition in Ref. [34]. As was stated in Ref. [23], 2\% isotropic hardening is the upper limit of many practical materials; it improves convergence, compared to an elastic perfectly plastic material model, with a small effect on the results (of less than $2.5 \%$ ).

The input parameter for the analysis was the displacement of the rigid flat in the axial direction, and all the corresponding results were obtained as output.

The convergence was tested by refining the mesh size until further refinement had negligible effect on the results. The accuracy of the finite element model was tested by comparison with the Hertz solution [18] for a homogenous sphere (identical coating and substrate materials) under the slip contact condition. The difference in the results was less than $1 \%$.

\section{Results and discussion}

The following typical input parameters were used for the numerical simulations: the radius of the spherical substrate varied in the range $2 \leq R \leq 12 \mathrm{~mm}$ and the dimensionless coating thickness $t / R$ was varied in the range $0.002 \leq t / R \leq 0.05$. The Young's moduli of the substrate and the coating materials varied in the range $200 \leq E_{\mathrm{su}} \leq 600 \mathrm{GPa}$ and $400 \leq E_{\mathrm{co}} \leq 2,000 \mathrm{GPa}$. The yield stresses of the substrate and the coating materials varied in the range $140 \leq Y_{\mathrm{su}} \leq 1000 \mathrm{MPa}$ and $285 \leq Y_{\mathrm{co}} \leq$ 3,300 MPa. The Poisson's ratios $v$ of the substrate and the coating were maintained equal and varied in the range $0.2<v<0.45$. This was done to cover a wide range of mechanical properties ratios, as follows: $2 \leq$ $E_{\mathrm{co}} / E_{\mathrm{su}} \leq 10$, which defines the mismatch between the coating and the substrate Young's modulus; $0.0007 \leq$ $Y_{\mathrm{su}} / E_{\mathrm{su}} \leq 0.0017$, which defines the substrate yield strain $\varepsilon_{\mathrm{y}_{-} \mathrm{su}}$; and $0.0007 \leq Y_{\mathrm{co}} / E_{\mathrm{co}} \leq 0.0017$, which defines the coating yield strain $\varepsilon_{\mathrm{y}_{-} \mathrm{co}}$.

In the following sections, the results obtained under the stick contact condition are discussed in comparison with the case of the slip contact condition, indicated as "slip" in all the figures.

\subsection{Yield inception}

Figure 6 presents the typical results for the dimensionless critical load $L_{\mathrm{c}} / P_{\mathrm{c}_{-} \text {co }}$ versus the dimensionless coating thickness, for various values of the Poisson's

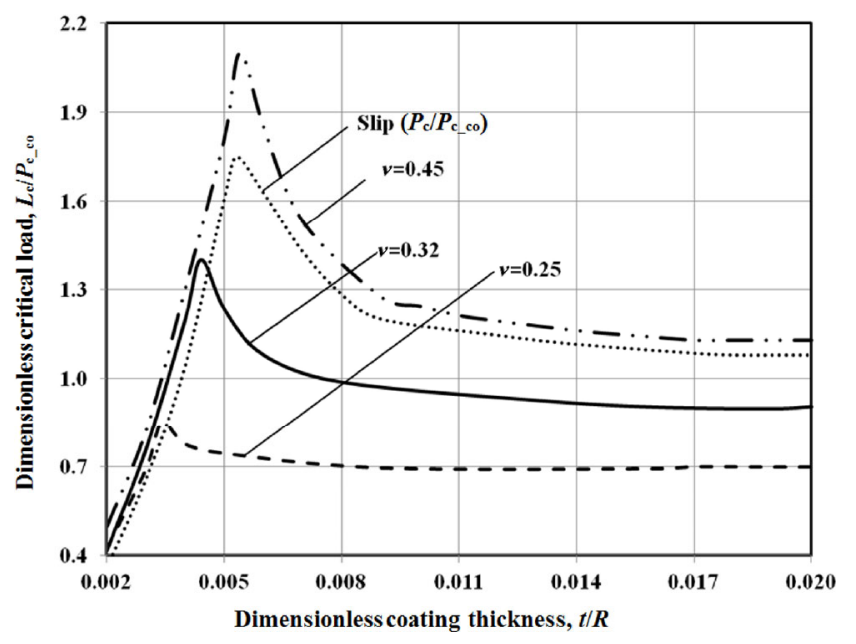

Fig. 6 Dimensionless critical load $L_{\mathrm{c}} / P_{\mathrm{c} \text { co }}$ vs. dimensionless thickness $t / R\left(E_{\mathrm{co}} / E_{\mathrm{su}}=4, \varepsilon_{\mathrm{y}_{\text {su }}}=\varepsilon_{\mathrm{y}_{-} \text {co }}=0.001\right)$.

ratio. The case indicated as "slip" presents the critical load $P_{\mathrm{c}} / P_{\mathrm{c}_{-} \text {co }}$ under the slip contact condition [25]. As can be seen, the general behavior under both stick and slip is similar, showing a maximum resistance to yield inception at a certain peak dimensionless thickness $(t / R)_{\mathrm{p}}$. It was found that under stick, like under slip, for coating thicknesses below $(t / R)_{p}$, the first yield inception always occurs in the substrate. As can be seen in Fig. 6, the effect of $v$ on $L_{\mathrm{c}}$ for such thicknesses is negligible. However, the peak values of $L_{c} / P_{c_{-} \text {co }}$ and their corresponding $(t / R)_{\mathrm{p}}$ increase with increasing Poisson's ratios. Also, for $t / R \geq(t / R)_{\mathrm{p}}$, where yield inception occurs in the coating, the critical load $L_{\mathrm{c}} / P_{\mathrm{c}_{-} \text {co }}$ at any given $t / R$ is higher for a larger Poisson's ratio. A similar behavior was also shown in Ref. [22] for a homogeneous sphere under the stick contact condition, where a larger Poisson's ratio results in lower tangential stresses in the contact area and a lower equivalent von Mises stress. For large coating thicknesses, the critical load approaches asymptotically the critical load in the case of a homogenous sphere made of the coating material. This case was analyzed in Ref. [22], and indeed Eq. (2) exactly fits the results shown in Fig. 6 for large $t / R$ values (see also $L_{\mathrm{c}} / P_{\mathrm{c}}$ in Fig. 2).

Figure 7 presents a comparison between the dimensionless von Mises stress $\sigma_{\mathrm{eq}} / Y$ along the axis of symmetry for stick and slip contact conditions at equal critical loads, which can be obtained at the intersection of the slip curve with any of the stick curves in Fig. 6. The results in Fig. 7 are for the case of $v=0.32$, 


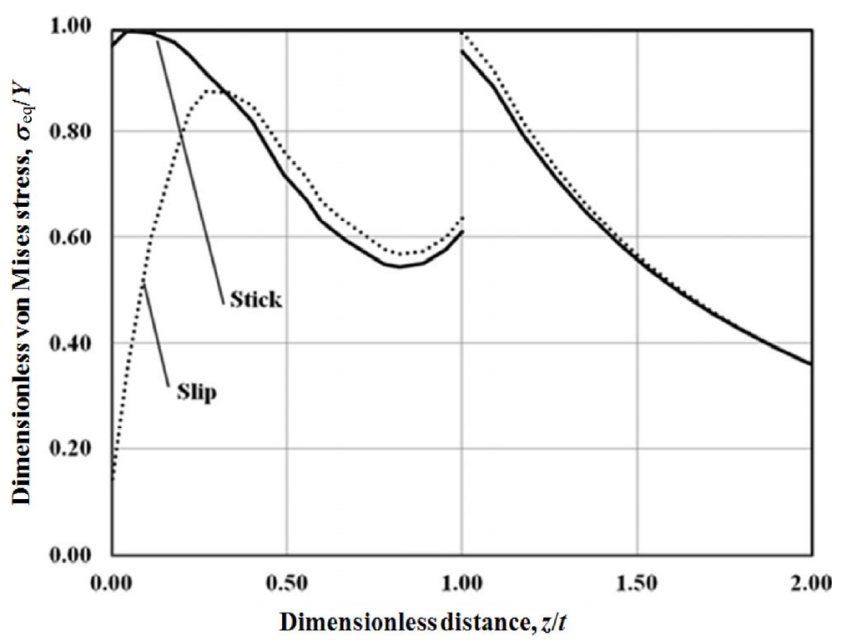

Fig. 7 Dimensionless von Mises equivalent stress along the axis of symmetry $\left(t / R=0.0045, E_{\mathrm{co}} / E_{\mathrm{su}}=4, \varepsilon_{\mathrm{y}_{\mathrm{S} s u}}=\varepsilon_{\mathrm{y}_{\mathrm{C}} \mathrm{co}}=0.001, \delta / R=\right.$ $\omega / R=1 \times 10^{-5}$ and $\left.v=0.32\right)$.

$t / R=0.0045$, and $\delta / R=\omega / R=1 \times 10^{-5}$. It should be noted here that this intersection occurs at $t / R>(t / R)_{\mathrm{p}}$ for the stick case but at $t / R<(t / R)_{\mathrm{p}}$ for the slip case. Hence, according to Ref. [25], the location of yield inception for the slip case is within the substrate. In Fig. 7, the results are presented against the dimensionless distance $z / t$, measured along the axis of symmetry from the center of the contact area. The coating/substrate interface is located at $z / t=1$, and the normalization of $\sigma_{\text {eq }}$ is done using the corresponding yield stress of the relevant material. As can be seen from the figure that the difference between the two contact conditions is apparent only close to the contact area, where the stress level under the stick contact condition is much higher than that under the slip contact condition. For the slip case, the yield inception $\left(\sigma_{\text {eq }} / Y=1\right)$ occurs at the substrate side of the interface, while for the stick case it occurs within the coating, slightly below the contact area.

Figure 8 presents the typical results for the effect of the Poisson's ratio under the stick contact condition, on $\sigma_{\mathrm{eq}} / Y$ along the axis of symmetry, for the same coating thickness $t / R=0.0045$ as in Fig. 7, but at a fixed dimensionless interference $\delta / R=\omega / R=6.5 \times 10^{-6}$ (different loads). For that interference, the case with $v=0.25$ reached the critical load while the other cases did not. Here again, like in Fig. 7, the difference between the various cases is apparent close to the contact area. The equivalent von Mises stress in this

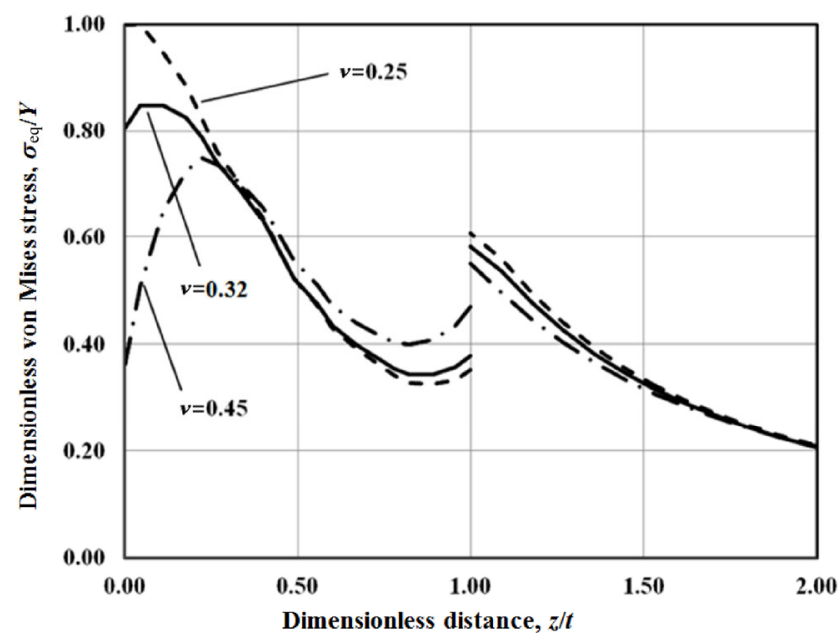

Fig. 8 Dimensionless von Mises equivalent stress along the axis of symmetry $\left(t / R=0.0045, E_{\mathrm{co}} / E_{\mathrm{su}}=4, \varepsilon_{\mathrm{y}_{-} \text {su }}=\varepsilon_{\mathrm{y}_{-} \text {co }}=0.001, \delta / R=\right.$ $\left.6.5 \times 10^{-6}\right)$.

region increases with decreasing Poisson's ratio, and at the same time its peak value occurs closer to the contact area, similar to the findings for a homogeneous sphere [22]. A higher peak value of $\sigma_{\text {eq }} / Y$ at a given interference indicates a smaller resistance to yield inception. This explains the increase in peak values of $L_{\mathrm{c}} / P_{\mathrm{c}_{\mathrm{c}} \mathrm{co}}$ with increasing Poisson's ratios, shown in Fig. 6.

By curve fitting the numerical results of $(t / R)_{p_{\text {_st }} \text { as }}$ as a function of $\varepsilon_{\mathrm{y}_{-} \mathrm{su}} \varepsilon_{\mathrm{y}_{-} \mathrm{co},}, E_{\mathrm{co}} / E_{\mathrm{su}}$ and Poisson's ratio over the entire range of the input parameters, the following empirical relation was obtained:

$$
\left(\frac{t}{R}\right)_{\text {p_st }}=3.95 v^{0.724}\left(\frac{E_{\mathrm{co}}}{E_{\mathrm{su}}}\right)^{0.39}\left(\varepsilon_{\mathrm{y}_{-} \mathrm{co}}\right)^{1.41}\left(\varepsilon_{\mathrm{y} \_ \text {su }}\right)^{-0.45}
$$

The $\left(R^{2}\right)$ goodness-of-fit for Eq. (13) was higher than 0.98. A comparison between Eq. (13) under the stick contact condition and Eq. (7) under the slip contact condition shows similarity in the structure, except for the dependence on Poisson's ratio under the stick contact condition.

Further investigation of the elastic-plastic regime under the stick contact condition was limited to dimensionless thicknesses $t / R>(t / R)_{p_{-} \text {st }}$ in order to ensure that the first yield inception will occur within the coating. This excludes unfavorable instances of possible failure owing to coating delamination or the weakening effect caused by yield inception at the coating/substrate interface or within the substrate. 
Normalizing the first critical interference $\delta_{\mathrm{c} 1}$ (for cases with first yield inception within the coating) by the critical interference $\omega_{\text {c_co }}$ of a homogeneous sphere made of the coating material (as was done in Ref. [34]), and curve fitting the numerical results, the following empirical dimensionless expression was obtained:

$$
\begin{aligned}
\frac{\delta_{\mathrm{c} 1}}{\omega_{\mathrm{c} \_ \text {co }}} & =\left[6.82 v-7.83\left(v^{2}+0.0586\right)\right] \\
& {\left[1+0.007\left(\frac{E_{\mathrm{co}}}{E_{\mathrm{su}}}-1\right)^{0.646}\left(\varepsilon_{\mathrm{y} \_ \text {co }}\right)^{0.244}\left(\frac{t}{R}\right)^{-1.21}\right] }
\end{aligned}
$$

The $\left(R^{2}\right)$ goodness-of-fit for Eq. (14) was higher than 0.93 . The first bracket of the equation presents the equation dependence of Poisson's ratio, while the second bracket presents the equation dependence of the geometrical and other material properties. In the first set of brackets, the dependence on the Poisson's ratio is identical to that for a homogeneous sphere $\left(E_{\mathrm{co}} / E_{\mathrm{su}}=1\right)$ under the stick contact condition (Eq. (1)). In the second set of brackets, it can be seen that when selecting the mechanical properties of a homogeneous sphere, the expression degenerates to 1 and Eq. (1) is obtained. Likewise, for large $t / R$, where the coated sphere behaves like a homogeneous sphere made from the coating material, the second set of brackets also degenerates to 1, and Eq. (1) is obtained again. Eq. (14) is independent of $\varepsilon_{y_{-} s u}$. This is because the substrate is still elastic in this regime and the only parameter influencing the substrate is $E_{\mathrm{su}}$.

When the interference increases above that corresponding to the first yield in the coating, $\delta_{\mathrm{c} 1}$, a second yield inception occurs in the substrate at a second critical interference $\delta_{\mathrm{c} 2}$ (similar to the behavior under the slip contact condition [34]). The empirical expressions obtained from the best fit of the numerical results for the interference, contact load, and contact area at the second yield inception are as follows:

$$
\begin{aligned}
\frac{\delta_{\mathrm{c} 2}}{\omega_{\mathrm{c} \_\mathrm{co}}} & =\left(\frac{t}{R}\right)^{1.17}\left(\frac{E_{\mathrm{co}}}{E_{\mathrm{su}}}-1\right)^{-0.09}\left(\varepsilon_{\mathrm{y}_{-} \mathrm{co}}\right)^{-1.93}\left(\varepsilon_{\mathrm{y} \_ \text {su }}\right)^{0.89} \\
\frac{L_{\mathrm{c} 2}}{P_{\mathrm{c} \_\mathrm{co}}} & =\left(\frac{t}{R}\right)^{1.8}\left(\frac{E_{\mathrm{co}}}{E_{\mathrm{su}}}-1\right)^{-0.27}\left(\varepsilon_{\mathrm{y}_{-} \mathrm{co}}\right)^{-2.3}\left(\varepsilon_{\mathrm{y} \_ \text {su }}\right)^{0.78}
\end{aligned}
$$

$$
\frac{A_{\mathrm{c}_{2} \_\mathrm{st}}}{A_{\mathrm{c} \_\mathrm{co}}}=\left(\frac{t}{R}\right)^{1.6}\left(\frac{E_{\mathrm{co}}}{E_{\mathrm{su}}}-1\right)^{-0.2}\left(\varepsilon_{\mathrm{y} \_ \text {co }}\right)^{-2}\left(\varepsilon_{\mathrm{y} \_ \text {su }}\right)^{0.7}
$$

The $\left(R^{2}\right)$ goodness-of-fit for Eqs. (15), (16), and (17) was higher than $0.97,0.95$, and 0.99 , respectively. As can be seen from the equations, the second critical parameters are independent of the Poisson's ratio (had less than $5 \%$ influence for the entire range of the Poison's ratios), and for a homogeneous sphere $\left(E_{\mathrm{co}} / E_{\mathrm{su}}=1\right)$ a second yield inception is unattainable (second yield parameters approach infinity). Simulations that were conducted under the slip contact condition, for the same mechanical parameters, showed very similar results for the second critical parameters. Hence, it can be concluded that for the entire range of input parameters in this study the effect of the contact condition on the second critical parameters is negligible.

\subsection{Plasticity evolution}

To better understand why the stick contact condition only affects the first yield inception in the coating and has a negligible effect on the second yield inception in the substrate, the plasticity boundaries under stick were compared to those under the slip contact condition for several special interferences, which are described in Ref. [34]. These special cases are: (1) the elastic core in the coating under the contact area completely disappears and a second yielding occurs on the substrate side of the interface; (2) reappearance of the elastic core under the contact area owing to increased plasticity in the substrate; (3) first appearance of a second elastic core in the coating; and (4) merging of the first and second elastic cores in the coating.

Figures 9 and 10 present the evolution of the plastic zones for a thick coating $t / R=0.05$ and two extreme cases of low and high mismatch of the coating and substrate moduli of elasticity, respectively. Each figure presents equal dimensional interferences in slip and stick, $\omega=\delta$. However, the dimensionless interferences $\omega / \omega_{\mathrm{c} 1}$ and $\delta / \delta_{\mathrm{c} 1}$ are different because $\omega_{\mathrm{c} 1} \neq \delta_{\mathrm{c} 1}$ (see Eqs. (8) and (14)). The solid and dashed lines in these figures represent the plastic boundaries for the stick and slip contact conditions, respectively. Hence, the zones within these boundaries are plastically deformed, while those outside are still elastic. As the finite element 
(a)

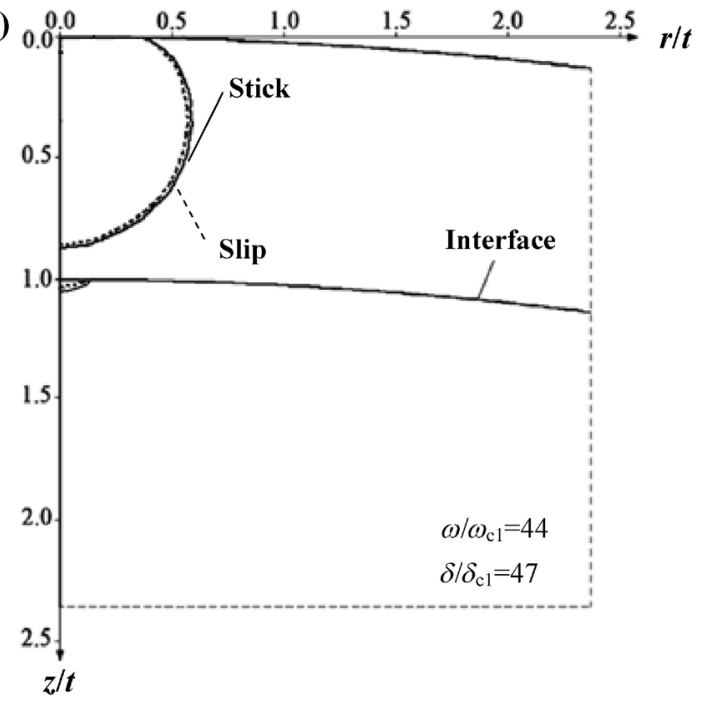

(b)

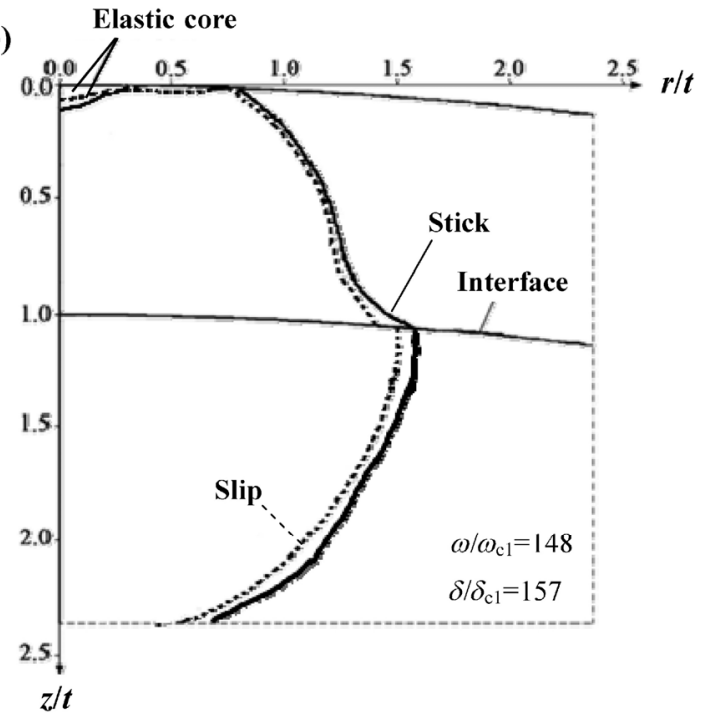

Fig. 9 Evolution of the plastic zones in the coated sphere $(v=$ $0.32, t / R=0.05, \varepsilon_{\mathrm{ysu}}=\varepsilon_{\mathrm{yco}}=0.001$ and $E_{\mathrm{co}} / E_{\mathrm{su}}=2$ ). (a) $\omega / \omega_{\mathrm{c} 1}=$ 44 and $\delta / \delta_{\mathrm{cl}}=47$ and (b) $\omega / \omega_{\mathrm{cl}}=148$ and $\delta / \delta_{\mathrm{cl}}=157$.

is axisymmetric, only half of the model is shown, and both axes are normalized using the coating thickness. In both cases, the Poisson's ratio is $v=0.32$. Figures 9(a) and $9(\mathrm{~b})$ present the two special cases (1) and (2) above, respectively. In Fig. 9(a), the plastic zones in the coating at $\omega / \omega_{\mathrm{c} 1}=44$ and $\delta / \delta_{\mathrm{c} 1}=47$ are almost identical for stick and slip. However, the evolution history (not shown in the figure) is very different. For slip, an elastic core was present under the contact area for lower interference, which vanished at $\omega / \omega_{\mathrm{c} 1}=$ 44. This elastic core did not appear in stick. Moreover, as shown in Fig. 9(a), the second yield inception in the substrate occurs simultaneously at $\omega / \omega_{\mathrm{c} 1}=44$ and

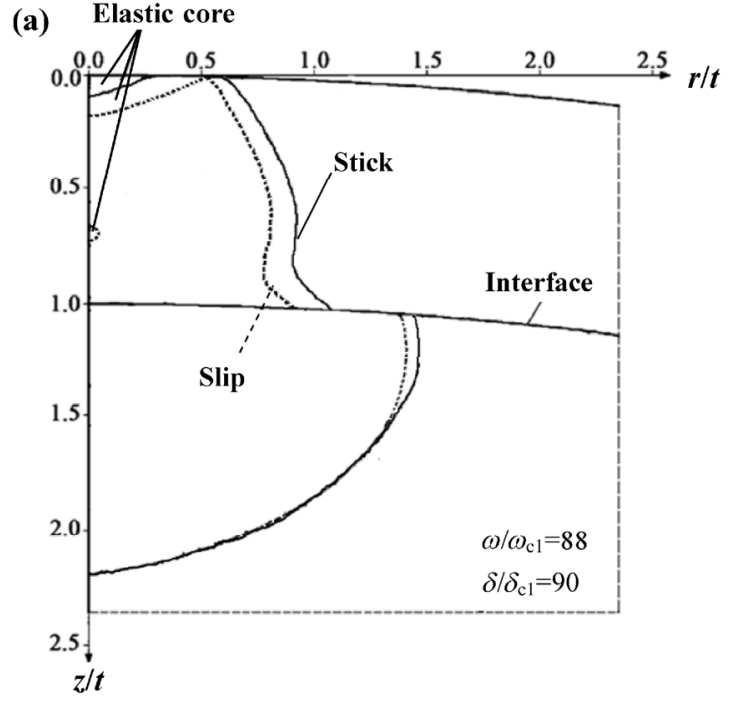

(b)

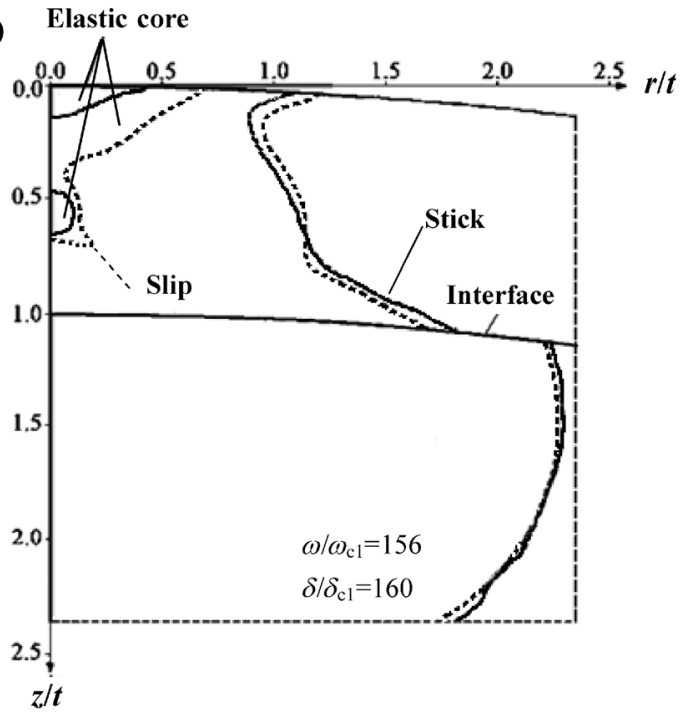

Fig. 10 Evolution of the plastic zones in the coated sphere ( $v=$ $0.32, t / R=0.05, \varepsilon_{\mathrm{y} \mathrm{su}}=\varepsilon_{\mathrm{y} \text { co }}=0.001$ and $\left.E_{\mathrm{co}} / E_{\mathrm{su}}=10\right)$. (a) $\omega / \omega_{\mathrm{c} 1}=$ 88 and $\delta / \delta_{\mathrm{c} 1}=90$ and (b) $\omega / \omega_{\mathrm{c} 1}=156$ and $\delta / \delta_{\mathrm{c} 1}=160$.

$\delta / \delta_{\mathrm{c} 1}=47$ for slip and stick, respectively. In Fig. 9(b), at $\omega / \omega_{\mathrm{c} 1}=148$ in slip and $\delta / \delta_{\mathrm{c} 1}=157$ in stick, the plastic zones in the coating and substrate for slip and stick also look similar. The main difference between the two cases is, once again, the elastic core in the coating under the contact area. In slip, this elastic core, which vanished at $\omega / \omega_{\mathrm{c} 1}=44$, reappears under the entire contact area at $\omega / \omega_{\mathrm{c} 1}=148$. In stick, at $\delta / \delta_{\mathrm{c} 1}=157$, the elastic core just appears for the first time. Figures 10(a) and $10(\mathrm{~b})$ present, respectively, the two other special cases, (3) and (4), indicated above. Here again, the plastic zones in the substrate are almost identical for slip and stick and the main difference is in the elastic 
cores within the coating. In Fig. 10(a), at $\omega / \omega_{\mathrm{c} 1}=88$ and $\delta / \delta_{\mathrm{c} 1}=90$, a second elastic core in the coating appears only in slip, and the first elastic core in stick is smaller than that in slip. In Fig. 10(b), at $\omega / \omega_{\mathrm{c} 1}=156$ and $\delta / \delta_{\mathrm{c} 1}=$ 160 , the two elastic cores in slip have merged while these in stick are still separated. A similar behavior to that shown in Figs. 9 and 10 was also observed when comparing the conditions of slip and stick for the case of thinner coating with $t / R=0.016$.

As can be seen, the contact condition has a significant effect on the plasticity evolution mainly within the coating close to the contact interface. The main difference that was observed is the behavior of the elastic cores for both contact conditions. This difference may be attributed to the additional tangential stresses at the contact area in stick.

\subsection{Contact load and contact area}

Figures 11 and 12 present typical dimensional results for the contact load $L$ and contact area $A_{\text {st }}$ respectively, versus the interference $\delta$. The individual effects of $E_{\mathrm{co}} / E_{\mathrm{su}}$ and $t / R$ are shown separately in parts (a) and (b), respectively. In each case the presented results are terminated at $\delta=\delta_{\mathrm{c} 2}$ (see Eq. (15)). As can be seen, different moduli ratios or coating thicknesses result in different load-interference and area-interference curves. In Fig. 11, it can be seen that at any given interference, increasing the moduli ratio or the coating thickness increases the contact load. Figure 12 shows that the contact area is almost linearly related to the interference. At any given interference, increasing the moduli ratio or decreasing the coating thickness decreases the contact area. This is similar to the results shown in Refs. [29, 30] for the slip contact condition in the elastic regime [29]. For large interferences $\left(\delta>\delta_{\mathrm{cl}}\right)$, varying the Poisson's ratio in the range $0.25<v<0.45$ had negligible effect (less than $8 \%$ ) on the results of the contact load and contact area. Normalizing the numerical results of the contact load, $L$, contact area, $A_{\mathrm{st}}$ and the interference, $\delta$, by their corresponding numerical second critical values $L_{\mathrm{c} 2}, A_{\mathrm{c} 2 \text { sts }}$ and $\delta_{\mathrm{c} 2}$ consolidated the different curves (various coating thicknesses $t / R$ and various $E_{\mathrm{co}} / E_{\mathrm{su}}$ ratios) in Figs. 11 and 12 into single curves, as can be seen in Fig. 13. The empirical relations for the dimensionless numerical
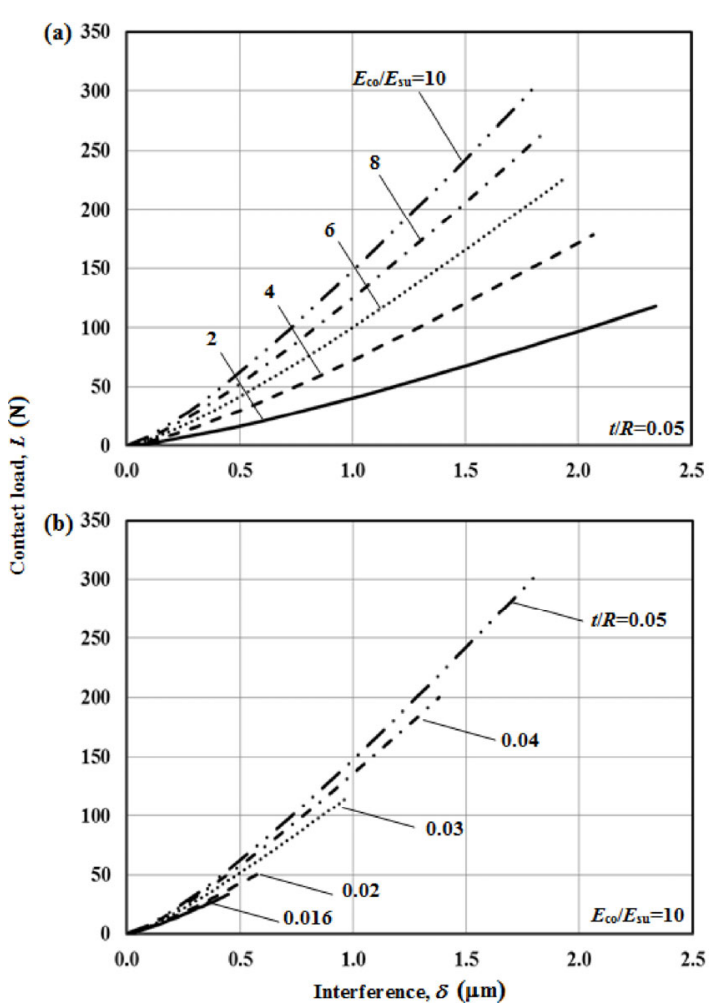

Fig. 11 Typical results for the contact load $L$ vs. the interference $\delta\left(\varepsilon_{\mathrm{y}_{-} \mathrm{su}}=\varepsilon_{\mathrm{y}_{\_} \text {co }}=0.001, v=0.32\right)$ : (a) $t / R=0.05$ and (b) $E_{\mathrm{co}} / E_{\mathrm{su}}=10$.

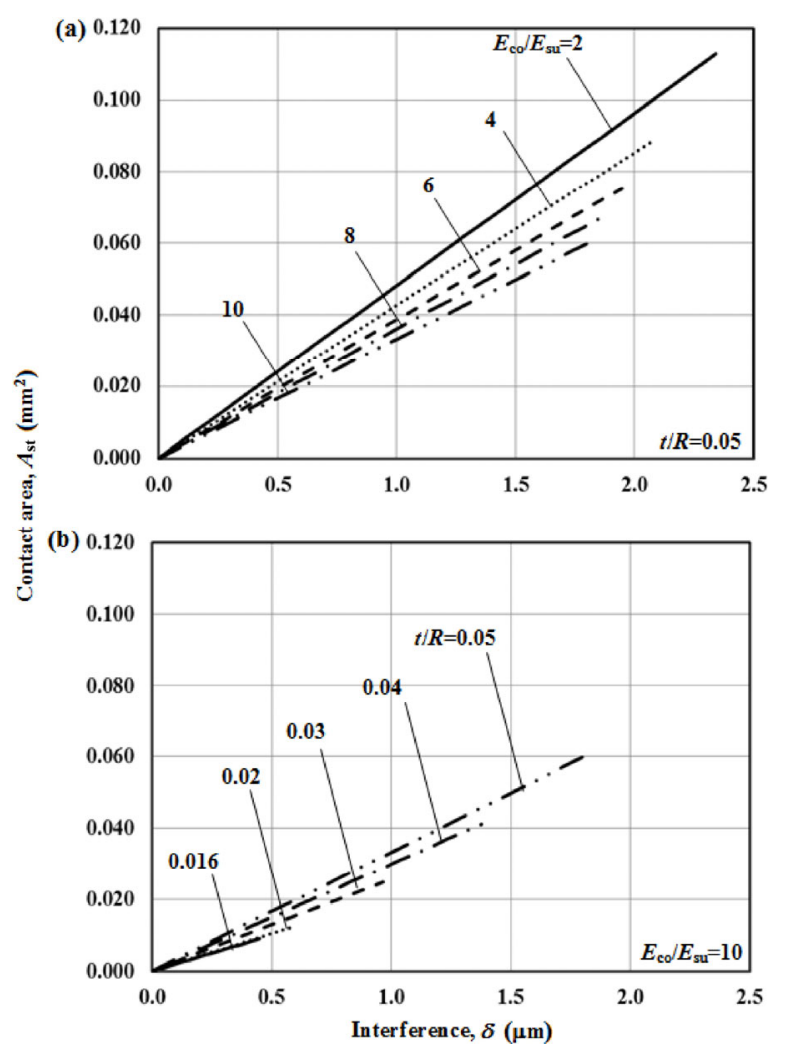

Fig. 12 Typical results for the contact area $A_{\text {st }}$ vs. the interference $\delta\left(\varepsilon_{\mathrm{y}_{\_} \text {su }}=\varepsilon_{\mathrm{y}_{-} \text {co }}=0.001, v=0.32\right):(\mathrm{a}) t / R=0.05$ and (b) $E_{\mathrm{co}} / E_{\mathrm{su}}=10$. 

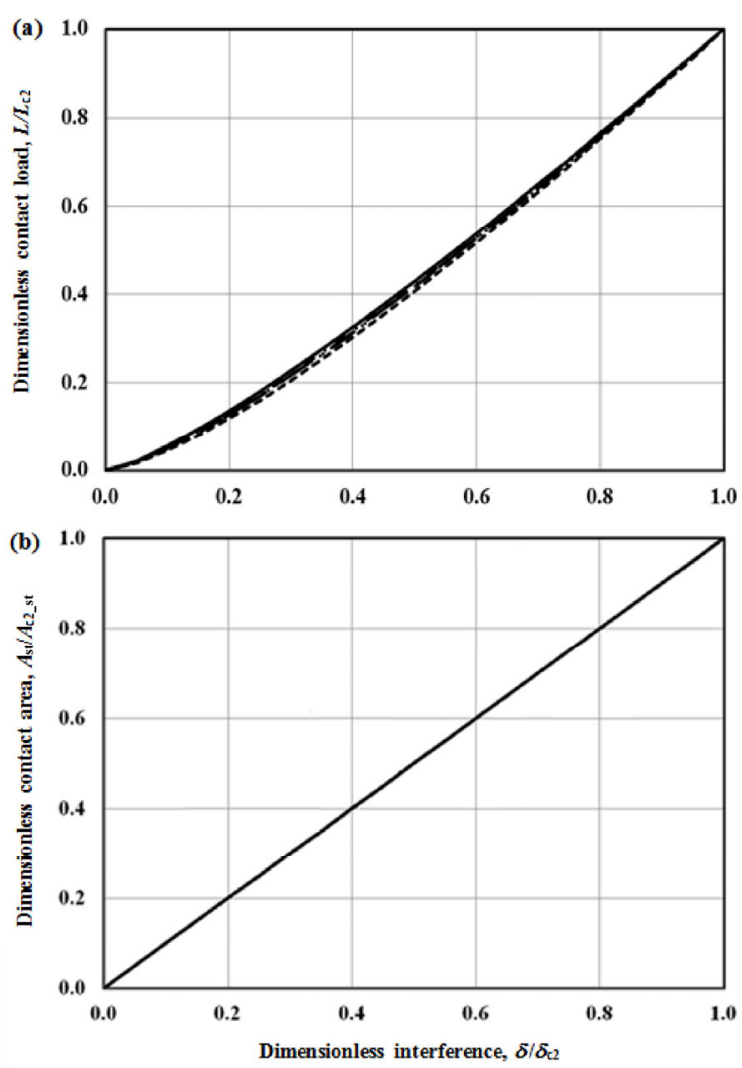

Fig. 13 (a) Dimensionless contact load $L / L_{\mathrm{c} 2}$ and (b) dimensionless contact area $A_{\mathrm{st}} / A_{\mathrm{c} 2 \_s t}$ vs. the dimensionless interference $\delta / \delta_{\mathrm{c} 2}$ for all the cases shown in Figs. 11 and 12.

results in the elastic-plastic regime for the range $\delta_{\mathrm{c} 1}<$ $\delta \leq \delta_{\mathrm{c} 2}$ are

$$
\begin{gathered}
\frac{L}{L_{\mathrm{c} 2}}=\left(\frac{\delta}{\delta_{\mathrm{c} 2}}\right)^{1.25} \\
\frac{A_{\mathrm{st}}}{A_{\mathrm{c} 2 \_\mathrm{st}}}=\frac{\delta}{\delta_{\mathrm{c} 2}}
\end{gathered}
$$

The $\left(R^{2}\right)$ goodness-of-fit for Eqs. (19) and (20) was better than 0.99. Simulations under the slip contact condition showed very similar results, indicating that the effect of contact condition on the contact parameters in the investigated elastic-plastic regime is negligible.

\section{Conclusion}

FEM was used to investigate the elastic-plastic contact of a coated sphere with a hard coating, compressed by a rigid flat under the stick contact condition. The effect of mechanical and geometrical properties on the contact parameters such as interference, load, and contact area was studied and compared with the corresponding results under the slip contact condition.

It was found that the general behavior of the onset of plasticity under stick and slip is similar, showing a maximum resistance to yield inception at a certain peak dimensionless thickness $(t / R)_{\mathrm{p}}$. The effect of the contact condition on the dimensionless contact parameters in the elastic-plastic regime, up to the second critical interference $\delta_{\mathrm{c} 2}$, is negligible except for the first critical interference $\delta_{\mathrm{c} 1}$ for coating thicknesses above $(t / R)_{p}$, when the first yield inception occurs within the coating slightly below the contact area. This is due to the additional tangential stresses at the contact area in stick.

The plasticity evolution under stick was studied and compared with that under the slip contact condition. The effect of the contact condition on the plastic zone boundaries is negligible, except for the evolution of elastic cores within the coating. This, too, may be attributed to the additional tangential stresses at the contact area in stick, which affect the stress level only within the coating close to the contact area.

The main purpose of studying the coated spherical contact under the stick condition is the ability to add a tangential loading, which cannot be supported under slip. Hence, this study is the first step towards developing a coated spherical contact under combined normal and tangential loading, which can be helpful in future research, to model the effect of coating properties on friction and wear. Substantial work is still needed in the future to consider additional parameters, and remove some of the simplified assumptions in the present analysis.

\section{Acknowledgment}

This paper is part of IEA AMT IA technical activities.

Open Access: The articles published in this journal are distributed under the terms of the Creative Commons Attribution 4.0 International License (http://creativecommons.org/licenses/by/4.0/), which permits unrestricted use, distribution, and reproduction in any medium, provided you give appropriate credit to the original author(s) and the source, provide a link 
to the Creative Commons license, and indicate if changes were made.

\section{References}

[1] Treutler C P O. Industrial use of plasma-deposited coatings for components of automotive fuel injection systems. Surface and Coatings Technology 200: 1969-1975 (2005)

[2] Scharf T W, Prasad S V, Dugger M T, Kotula P G, Goeke R S, Grubbs R K. Growth, structure, and tribological behavior of atomic layer-deposited tungsten disulphide solid lubricant coatings with applications to MEMS. Acta Materialia 54: 4731-4743 (2006)

[3] Law H H, Holden C A, Sapjeta J, Crane G R, Nakahara S. Electrical contact phenomena of nickel electrodeposits with sharp micro-asperities. Transactions on Components Hybrids and Manufacturing Technology 14: 585-591 (1991)

[4] Neubauer E, Korb G, Eisenmenger-Sittner C, Bangert H, Chotikaprakhan S, Dietzel D, Mansanares A M, Bein B K. The influence of mechanical adhesion of copper coatings on carbon surfaces on the interfacial thermal contact resistance. Thin Solid Films 433: 160-165 (2003)

[5] Clarke D R. Materials selection guidelines for low thermal conductivity thermal barrier coatings. Surface and Coatings Technology 163: 67-74 (2003)

[6] Piazza F, Grambole D, Schneider D, Casiraghi C, Ferrari A C, Robertson J. Protective diamond-like carbon coatings for future optical storage disks. Diamond and Related Materials 14: 994-999 (2005)

[7] Schintlmeister W, Wallgram W, Kanz J, Gigl K. Cutting tool materials coated by chemical vapor deposition. Wear 100: 153-169 (1984)

[8] Greenwood J, Williamson J. Contact of nominally flat surfaces. The Royal Society (London) 295: 300-319 (1966)

[9] Chang W, Etsion I, Bogy D B. An elastic-plastic model for the contact of rough surfaces. ASME Journal of Tribology 109: 257-263 (1987)

[10] Jackson R L, Kogut L. A comparison of flattening and indentation approaches for contact mechanics modeling of single asperity contacts. Tribology Transactions 128: 209-212 (2006)

[11] Komvopoulos K. Finite element analysis of a layered elastic solid in normal contact with a rigid surface. ASME Journal of Tribology 110: 477-485 (1988)

[12] Kral E, Komvopoulos K, Bogy D. Finite element analysis of repeated indentation of an elastic-plastic layered medium by a rigid sphere, Part II: Subsurface results. Journal of Applied Mechanics 62: 29-42 (1995)
[13] Ronkainen H, Koskinen J, Varjus S, Holmberg K. Loadcarrying capacity evaluation of coating/substrate systems for hydrogen-free and hydrogenated diamond-like carbon films. Tribology Letters 6: 63-73 (1999)

[14] Ye N, Komvopoulos K. Indentation analysis of elastic-plastic homogeneous and layered media: criteria for determining the real material hardness. ASME Journal of Tribology 125 : 685-691 (2003)

[15] Botelho T D S, Progri R, Inglebert G, Robbe-Valloire F. Analytical and experimental elastoplastic spherical indentations of a layered half-space. Mechanics of Materials 40: 771-779 (2008)

[16] Ahmadi S R, Shakeri M, Sadough A. Stress analysis in thin coatings and substrate subjected to point contact loading (mechanics of scratch test). Journal of Materials Processing Technology 205: 89-98 (2008)

[17] Kulchytsky-Zhyhailo R, Rogowski G. Stresses in hard coating due to a rigid spherical indenter on a layered elastic half-space. Tribology International 43: 1592-1601 (2010)

[18] Johnson K L. Contact Mechanics 1st ed. Cambridge: Cambridge University Press, 1985.

[19] Kogut L, Etsion I. Elastic-plastic contact analysis of a sphere and a rigid flat. Journal of Applied Mechanics 69: 657-662 (2002)

[20] Goodman L E. Contact stress analysis of normally loaded rough spheres. Journal of Applied Mechanics 29: 515-522 (1962)

[21] Spence D A. The Hertz contact problem with finite friction. Journal of Elasticity 5(3-4): 297-319 (1975)

[22] Brizmer V, Kligerman Y, Etsion I. The effect of contact conditions and material properties on the elasticity terminus of a spherical contact. International Journal of Solids and Structures 43: 5736-5749 (2006)

[23] Brizmer V, Zait Y, Kligerman Y, Etsion I. The effect of contact conditions and material properties on elastic-plastic spherical contact. Journal of Mechanics of Material and Structures 1(5): 865-879 (2006)

[24] Overcharenko A, Hlperin G, Verberne G, Etsion I. In situ investigation of the contact area in elastic-plastic spherical contact during loading-unloading. Tribology Letters 25(2): 153-160 (2007)

[25] Goltsberg R, Etsion I, Davidi G. The onset of plastic yielding in a coated sphere compressed by a rigid flat. Wear 271: 2968-2977 (2011)

[26] Song W, Li L, Ovcharenko A, Jia D, Etsion I, Talke F E. Plastic yield inception of an indented coated flat and comparison with a flattened coated sphere. Tribology International 52: 61-67 (2012) 
[27] Huang X, Kasem H, Shang H F, Shao T M, Etsion I. Experimental study of a potential weakening effect in spheres with thin hard coatings. Wear 296: 590-597 (2012)

[28] Goltsberg R, Etsion I. A model for the weakening effect of very thin hard coatings. Wear 308: 10-16 (2013)

[29] Goltsberg R, Etsion I. A universal model for the loaddisplacement relation in an elastic coated spherical contact. Wear 322: 126-132 (2015)

[30] Goltsberg R, Etsion I. Contact area and maximum equivalent stress in elastic spherical contact with hard coating. Tribology International 93: 289-296 (2016)

[31] Sun Y, Bloyce A, Bell T. Finite element analysis of plastic deformation of various TiN coating/substrate systems under

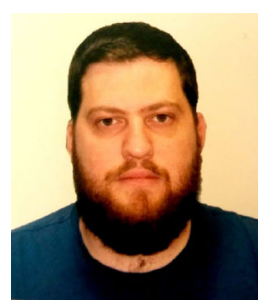

Shai RONEN. He received his bachelor degree in mechanical engineering in 2010 from Technion-

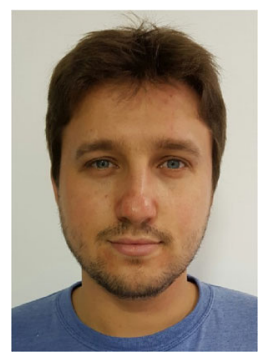

Roman GOLTSBERG. He received his bachelor degree in mechanical engineering in 2010 from TechnionIsrael Institute of Technology. In

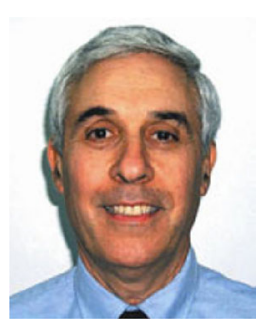

Izhak ETSION. He received his $\mathrm{PhD}$ degree in 1974 from Technion-Israel Institute of Technology. Since then he was a faculty at the Department of Mechanical Engineering where he is currently a Professor Emeritus. His research interests are in hydrodynamic lubrication, surface texturing, contact mechanics, nano-tribology and bio-tribology. Etsion normal contact with a rigid sphere. Thin Solid Films 271: 122-131 (1995)

[32] Kral E, Komvopoulos K, Bogy D. Finite element analysis of repeated indentation of an elastic-plastic layered medium by a rigid sphere, Part II: Subsurface results. Journal of Applied Mechanics 62: 29-42 (1995)

[33] Yoo Y-H, Lee W, Shin H. Spherical nano-indentation of a hard thin film/soft substrate layered system: II. Evolution of stress and strain fields. Modeling and Simulation in Materials Science and Engineering 12: 69-78 (2004)

[34] Chen Z, Goltsberg R, Etsion I. Plasticity evolution in a coated sphere compressed by a rigid flat. Tribology International 98: 116-124 (2016)

Israel Institute of Technology. Recently he had obtained his master degree in design and manufacturing management from the same institute.

2015 he had obtained his Ph.D. degree in mechanical engineering from the same institute. His research interests include contact mechanics of coatings and hydrodynamic bearings.

has published over 200 papers on various aspects of tribology and holds 15 patents. He is the founder of Surface Technologies Ltd. that developed the laser surface texturing (LST) technology for friction and wear reduction. His honors include Fellow of the ASME (1999), Fellow of the STLE (2001), the STLE 2005 International Award and the ASME 2016 Mayo D. Hersey Award. 